Europhys. Lett., 55 (3), pp. 317-323 (2001)

\title{
Effect of order parameter fluctuations on the Halperin-Lubensky-Ma first-order transition in superconductors and liquid crystals
}

\author{
I. F. Herbut ${ }^{1}$, A. Yethiraj ${ }^{2}$ and J. Bechhoefer ${ }^{1}$ \\ 1 Department of Physics, Simon Fraser University \\ Burnaby, British Columbia, Canada V5A $1 S 6$ \\ 2 Physics Department, University of Utrecht, and FOM Institute \\ for Atomic and Molecular Physics - The Netherlands
}

(received 28 November 2000; accepted in final form 18 May 2001)

PACS. 05.70.Fh - Phase transitions: general studies.

PACS. 05.70.Jk - Critical point phenomena.

PACS. 64.70.Md - Transitions in liquid crystals.

\begin{abstract}
We show that order parameter fluctuations in a good type-I superconductor or a liquid crystal always increase the size of the first-order transition. This behavior is eventually changed when the system crosses over to an inverted- $X Y$ critical behavior, with the size of the first-order transition vanishing as a power law with a crossover exponent. We find good agreement between our theory and a recent experiment on the nematic-smectic-A first-order transition in 8CB-10CB mixtures of liquid crystals.
\end{abstract}

More than 25 years ago, Halperin, Lubensky and Ma (HLM) [1] and Coleman and Weinberg [2] demonstrated that when a scalar field is coupled to a gauge field, the fluctuations of the gauge field can change the nature of the phase transition in the theory from continuous to first order. The coupling of a scalar field (or an order parameter (OP)) to a massless gauge field arises often in physics: the Meissner transition in superconductors [3], the Higgs mechanism in particle physics [4], and the nematic-smectic-A transition in liquid crystals [5] are among the best-known examples $[6,7]$. The analysis of HLM initially centered on the fluctuations of the gauge field and neglected the OP fluctuations, which is justifiable for good type-I superconductors (with Ginzburg-Landau parameter $\kappa \ll 1$ ), where the size (here defined as the difference between the transition and the critical temperatures) of the first-order transition is larger. This approximation, however, inevitably breaks down for strong type-II materials $(\kappa \gg 1)$, where neglecting the OP fluctuations would yield a first-order transition well into the critical region. Close to four dimensions, the effect of the OP fluctuations can be studied using the Wilson-Fisher renormalization group [1], which, however, in this case leads only to "run-away" flows and no stable critical points. This is usually interpreted as a sign of a first-order transition [1,8]. Today, based on accumulated analytical and numerical evidence, it is generally believed that the transition for $\kappa \gg 1$ is again second order, in the so-called inverted- $X Y$ universality class [9-17]. The corresponding topology of the flow of the 


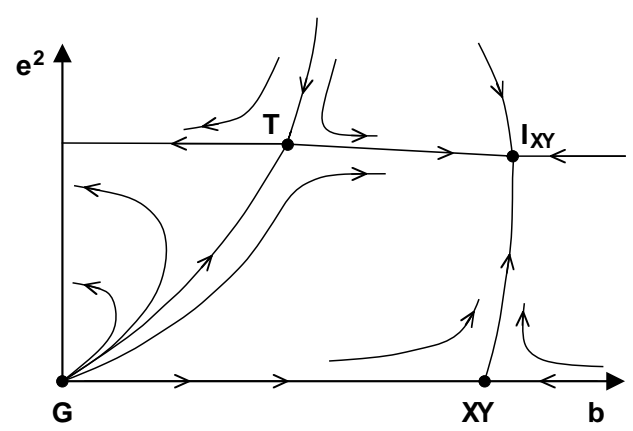

Fig. 1 - Schematic flow diagram of the quartic coupling $b$ and the charge $e^{2}$ (see eq. (1)) at the critical surface $T=T^{*}$. Note the Gaussian $(G), X Y$, inverted- $X Y\left(I_{X Y}\right)$, and the tricritical $(T)$ fixed points. Good type-I materials $(\kappa \ll 1)$ lie far to the left of the separatrix that connects the Gaussian and the tricritical fixed points.

coupling constants under scaling transformation is depicted in fig. 1. It may thus seem natural to expect that the OP fluctuations should decrease the size of the first-order transition, finally reducing it to zero at the crossover to an inverted- $X Y$ critical behavior.

The fluctuation effects in question are unfortunately too fine to be observable even in high$T_{\mathrm{c}}$ superconductors on account of the smallness of the fine-structure constant $[18,19]$. But in liquid crystals, the coupling of the smectic OP to the director fluctuations is stronger, and the fluctuations at the nematic-smectic-A transition become an issue of central importance. A recent experiment on two-component liquid-crystal mixtures [20] found a surprising result: the size of the first-order transition is larger than the prediction of the HLM theory. Motivated by this result, in this letter we consider theoretically the effect of OP fluctuations on the first-order transition in a type-I material. We show quite generally that for a small enough Ginzburg-Landau parameter $\kappa$, the size of the first-order transition is indeed always larger than the HLM result. Crudely, the reason is that for a good type-I material, the effective Ginzburg-Landau parameter $\kappa$ always decreases at large scales, making the material only more type I and the transition more strongly first order. The type-I region is defined here as left of the separatrix in fig. 1, where the flow is qualitatively the same as that of HLM near four dimensions. This behavior is eventually changed as the separatrix between type-I (first-order) and type-II (inverted- $X Y$ ) regimes is approached, where the size of the transition goes to zero as a power law, with a crossover exponent that characterizes the tricritical point. Using the simple one-loop recursion relations for the coupling constants in an isotropic version of the Ginzburg-Landau-Wilson action, we show that the experimental data of Yethiraj and Bechhoefer are fit rather well by our theory.

We are interested in a general phase transition described by the three-dimensional GinzburgLandau-Wilson theory for a fluctuating complex OP $\Psi(\vec{r})$ minimally coupled to a fluctuating vector potential (also known as the Higgs scalar electrodynamics):

$H=\int \mathrm{d}^{3} \vec{r}\left[|(\nabla-i e \vec{A}(\vec{r})) \Psi(\vec{r})|^{2}+\frac{a(T)}{2}|\Psi(\vec{r})|^{2}+\frac{b}{2}|\Psi(\vec{r})|^{4}+\frac{c}{6}|\Psi(\vec{r})|^{6}+\frac{1}{2}(\nabla \times \vec{A}(\vec{r}))^{2}\right]$,

where $a(T)=\alpha\left(T-T_{0}\right) / T_{0}$, and we assume the superconducting gauge, $\nabla \cdot \vec{A}=0$. For simplicity, we neglect the anisotropy inherent to liquid crystals, but leave the sixth-order term in the Ginzburg-Landau expansion. Fields and lengths have been chosen so that the number 
of couplings in the theory is minimal. HLM showed that neglecting the fluctuations of the OP and integrating out the vector field $\vec{A}$ around the uniform OP configuration $\Psi_{0}$, to the lowest order in charge $e$ one obtains the corrected mean-field Ginzburg-Landau free energy per unit volume:

$$
F=\frac{a(T)}{2} \Psi_{0}^{2}-\frac{e^{3} \sqrt{2}}{3 \pi} \Psi_{0}^{3}+\frac{b}{2} \Psi_{0}^{4}+\frac{c}{6} \Psi_{0}^{6}
$$

in units where $k_{\mathrm{B}} T_{0}=1$. The negative cubic term in the free energy then implies a firstorder transition. A useful measure of the size of the first-order transition is the parameter $t=\left(T_{\mathrm{c}}-T^{*}\right) / T^{*}$, where $T_{\mathrm{c}}$ is the first-order transition temperature, and $T^{*}$ the spinodal point at which the normal phase becomes unstable. First, to demonstrate our main point of principle and keep the algebra simple, we assume $b>0$ and temporarily set $c=0$ in eq. (2). Later, when we attempt a quantitative comparison with the experiment we will turn $c$ back to a finite value. When $c=0$, from eq. (2), the size of the transition is

$$
\alpha t_{\mathrm{HLM}}=\frac{e^{4}}{(3 \pi)^{2}} F(\kappa),
$$

where $\kappa^{2}=b / 2 e^{2}$ is the dimensionless Ginzburg-Landau parameter, and $F(\kappa)=1 / \kappa^{2}$. For $\kappa \ll 1$, the first-order transition thus occurs at higher temperatures, where the OP fluctuations may indeed be neglected. The HLM result becomes asymptotically correct in this limit.

To include the OP fluctuations, assume that the first-order transition in the theory (eq. (1)) with the coupling constants $e$ and $b$ occurs at some $t \neq t_{\mathrm{HLM}}$. In general, the renormalizability of the theory (eq. (1)) implies that rescaling the cutoff $\Lambda \rightarrow \Lambda / s$ by an arbitrary factor $s>1$ is equivalent to changing the coupling constants into $e(s)$ and $b(s)$, with the first-order transition for these renormalized couplings occurring at some new temperature $t_{0}$. The variation of the renormalized couplings $e(s), b(s)$, and the reduced temperature $t(s)$ with $s$ is described by differential recursion relations of the form

$$
\frac{\mathrm{d} \lambda(s)}{\mathrm{d} \ln (s)}=\beta_{\lambda}\left[e^{2}(s), b(s), t(s)\right]
$$

where $\lambda=\left\{e^{2}, b, t\right\}$. If $t_{0}$ is large enough, the OP fluctuations at the first-order transition in the rescaled theory indeed become negligible, and $t_{0}$ may be approximated by the mean-field (HLM) expression:

$$
\alpha t_{0} \approx \frac{e^{4}(s)}{(6 \pi)^{2}} F(\kappa(s)) .
$$

Together with eqs. (3) and (4), and the boundary conditions $t(1)=t$ and $t(s)=t_{0}$, eq. (5) determines implicitly the actual size of the transition $t$. Clearly, the above idea is quite general and applicable to other weakly first-order transitions. First, let us demonstrate that neglecting the interactions between fluctuations by renormalizing the coupling constants only according to dimensional analysis gives just the HLM result. Power counting in eq. (1) implies that $t(s)=t s^{2}, e^{2}(s)=e^{2} s, b(s)=b s$. Thus, $\kappa(s)=\kappa$, and dividing eqs. (3) and (5) gives $t=t_{\mathrm{HLM}}$. More generally, for small $e^{2}$ and $b$, and for $t \ll e^{2}, b$, the renormalized couplings obey the (one-loop) differential equations (eq. (4)) with $[1,11]$

$$
\begin{aligned}
& \beta_{t}=t\left(2+u e^{2}-v b+\mathcal{O}\left(e^{4}, b^{2}, b e^{2}\right)\right), \\
& \beta_{e}=e^{2}-x e^{4}+\mathcal{O}\left(e^{6}\right), \\
& \beta_{b}=b-y b^{2}+z b e^{2}-w e^{4}+\mathcal{O}\left(b^{3}, e^{6}, b e^{4}, b^{2} e^{2}\right) .
\end{aligned}
$$


The signs in the above equations are chosen so that the numerical coefficients $u, v, x, y, z$ and $w$ are positive $[1,11]$. The differential recursion relation for the Ginzburg-Landau parameter $\kappa$, for $\kappa \ll 1$, may be easily obtained from eqs. (7) and (8):

$$
\frac{\mathrm{d} \kappa^{2}}{\mathrm{~d} \ln (s)}=-\frac{w}{2} e^{2}+\mathcal{O}\left(e^{4}, e^{2} \kappa^{2}\right) .
$$

Note that the right-hand side in the last equation is negative, and $\kappa$, if initially small enough, always decreases under renormalization. This is a consequence of the $e^{4}$ term in eq. (8), which generates a negative quartic coupling and tends to drive the transition first order. The same term can be seen to be responsible for the runaway flows near four dimensions in the original HLM analysis. For small charge $e$, one may neglect the nonlinear terms and solve eqs. (6) and (7) to find, for small $\kappa$,

$$
\frac{t}{t_{\mathrm{HLM}}}=\frac{\kappa^{2}}{\kappa^{2}\left(\sqrt{t_{0} / t}\right)} .
$$

Since $\kappa(s)<\kappa$, the solution to the above equation always gives $t>t_{\mathrm{HLM}}$ for a good type-I material. In sum, while near the Gaussian fixed point $t$ and $e^{2}$ approximately scale according to dimensional analysis, a small Ginzburg-Landau parameter $\kappa$ acquires a small negative dimension and renormalizes downwards, thus increasing the size of the first-order transition.

Assuming the general topology of the flow diagram as shown in fig. 1, the size of the firstorder transition will eventually go to zero as the coupling constants approach the separatrix between type-I and type-II regimes. If the separatrix at the critical surface $t=0$ lies at some $b_{c}(e)$, close to the separatrix one finds

$$
t \propto\left[b_{c}(e)-b\right]^{1 / \phi},
$$

where $\phi=r \nu$ is the crossover exponent, with $\nu^{-1}=\partial \beta_{t} / \partial t$, taken at the tricritical fixed point. Here, $r$ is the (positive) scaling dimension of the second relevant scaling variable at the tricritical point, which in general is a linear combination of $e^{2}, b$, and $t$. Near four dimensions, the result in eq. (11) agrees with that of HLM: the transition is then always first order, unless $e=0$. The role of the tricritical point near four dimensions is played by the $X Y$ fixed point, which has two relevant directions, $t$ and $e^{2}$, with $\nu=\nu_{X Y}$ and $r=\varepsilon$, where $\varepsilon=4-d$. Equation (11) then becomes $t \propto e^{2 /\left(\varepsilon \nu_{X Y}\right)}$, which is just eq. (17) in $[1,21]$.

By tuning the concentration $x$ of $10 \mathrm{CB}$ liquid crystals in $8 \mathrm{CB}$, one can vary the temperature range of the nematic phase, and, in effect, tune the parameter $b$ in eq. (1). For larger concentrations, $b$ is negative and the transition is more strongly first order. The latent-heat data for $x>0.42$ are well fit by mean-field theory (eq. (2)) with the parameters $c=1$, $\alpha=3.35, b=b_{0}\left(x^{*}-x\right)$, with $b_{0}=0.395$ and $x^{*}=0.42$, and $e^{2}=0.0421$ [22-24]. For $x<x^{*}$, the quartic term in eq. (2) becomes positive, but the transition remains first order, in agreement with the HLM theory. At smaller concentrations, $t$ continues to decrease, as expected, but there is a clear deviation from the HLM result [20]. To attempt to fit the data in the whole concentration range $0<x<0.65$, we take the above parameters to set the initial values of the couplings, and then evolve $t, e$, and $b$ according to eqs. (6)-(8), with $u=v=1 / 4, x=1 / 16, y=(2 \sqrt{2}+1) / 8, z=1 / 2$, and $w=1 /(2 \sqrt{2})$, which is the result of the standard field-theoretic calculation in three dimensions [11]. We neglect the change of $c$ under scaling, since the results are quite insensitive to its precise value. The scaling of the couplings is stopped at $s=S$ at which

$$
\alpha t_{0}=\frac{e^{3}(S) \sqrt{2}}{\pi} \Psi_{0}-2 b(S) \Psi_{0}^{2}-c \Psi_{0}^{4},
$$




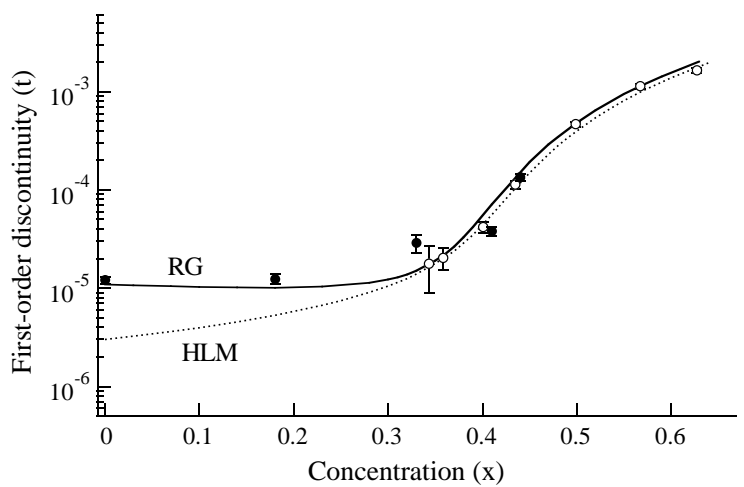

Fig. 2 - Size of the first-order NA transition $(t)$ as a function of $10 \mathrm{CB}$ concentration $(x)$ in the $8 \mathrm{CB}-$ 10CB system. Comparison between the RG result (full line), HLM (dashed line), and experiment. Filled circles are data from Yethiraj and Bechhoefer, while hollow circles are latent-heat data from Marynissen et al., converted into equivalent size $t$.

where $\Psi_{0}$ is determined by the equation

$$
\frac{2 c}{3} \Psi_{0}^{3}+b(S) \Psi_{0}-\frac{\sqrt{2}}{3 \pi} e^{3}(S)=0 .
$$

These are the standard mean-field equations for the first-order transition that follow from eq. (2). (For $c=0$, eqs. (12), (13) imply eq. (3)). By neglecting fluctuations at the scale $S$ set by eqs. (12), (13) we assume that $t_{0}$ is large enough so that the mean-field HLM theory essentially correctly predicts the size of the transition. The only free parameter left then is the value of $t_{0}$. We chose $t_{0}=0.01$, an order of magnitude larger than the largest measured $t$ in the experiment. The quality of the fit turns out not to be critically dependent on this choice. The size of the transition is thus determined by the initial condition $t(s=1)$ that satisfies eqs. (12), (13) at $s=S$. The result of the calculations and the comparison with HLM is shown in fig. 2. Note that for $x>0.35$, there is very little change from the HLM result [25], but as $b$ increases at smaller concentrations, deviations from HLM become significant, in agreement with the experiment. One expects that if one could increase $b$ further, eventually $t$ would go to zero in accord with eq. (11). The full nonperturbative structure of the flow diagram in fig. 1, with the tricritical and the inverted- $X Y$ fixed points, however, is beyond the simple one-loop $\beta$-functions we used [26]. The data suggest that, at small charge, the tricritical point is likely to lie at some $\kappa_{\mathrm{c}}>\sqrt{2}$, which corresponds approximately to the smallest concentration used in the experiment $(x=0)$.

Although anisotropy is known to be important in liquid crystals [5], its inclusion, besides introducing two new couplings, does not essentially change the structure of eqs. (6)-(8) [27]. In particular, the sign of the $e^{4}$-term in eq. (8) stays negative, and our main point that the size of the transition in good type-I materials is larger than the mean-field prediction remains valid. Some small quantitative differences from our result would be expected, however.

Calculations similar to ours have been performed in the past [21] near four dimensions, where there are no tricritical and critical points. Although the focus of that study was primarily the extreme type-II regime, the results are in qualitative agreement with ours.

Finally, the magnetic field can be shown to add a mass to the gauge field in eq. (1) [20], and therefore decrease the size of the first-order transition. A preliminary calculation (similar to the one in ref. [13]) of the recursion relation for the small magnetic field indicates that the 
OP fluctuations tend to increase the value of the critical field at which the transition becomes continuous, in a qualitative agreement with experiment [20]. A more quantitative analysis is left for future work.

In conclusion, we have shown that the naive expectation that order parameter fluctuations would always decrease the strength of the first-order transition predicted by Halperin, Lubensky, and Ma is incorrect for good type-I materials. While the transition size does decrease monotonically as the material becomes more type II, it becomes smaller than the HLM prediction only near the tricritical point that separates type-I and type-II regimes. By using one-loop renormalization group to calculate the evolution of Landau coefficients in the free energy, we can account for the deviations from HLM predictions that were observed in a recent experiment.

Our arguments also lead one to expect a nontrivial exponent near the tricritical point where the transition eventually should become second order. One would expect this point to be accessible experimentally. For example, one can view the variation of concentration $x$ in the $8 \mathrm{CB}-10 \mathrm{CB}$ experiments as a way of continuously adjusting the effective molecular length (between 8 and $10 \mathrm{CH}_{2}$ lengths). Mixing $8 \mathrm{CB}$ with a shorter molecule (for example, 6CB [28]) might then give access to the tricritical point and allow at least a crude measurement of the crossover exponent $\phi$.

$$
* * *
$$

This work has been supported by NSERC (Canada). IFH was also supported by an award from Research Corporation.

\section{REFERENCES}

[1] Halperin B. I., Lubensky T. C. and Ma S. K., Phys. Rev. Lett., 32 (1974) 292.

[2] Coleman S. and Weinberg E., Phys. Rev. D, 7 (1973) 1988.

[3] Ginzburg V. L. and Landau L. D., Zh. Eksp. Teor. Fiz., 20 (1950) 1064.

[4] Higgs P. W., Phys. Lett., 12 (1964) 132.

[5] De Gennes P. G. and Prost J., The Physics of Liquid Crystals, 2nd edition (Clarendon Press, Oxford) 1993, and references therein.

[6] For applications to other condensed matter systems, see, for example, Wen X. G. and Wu Y. S., Phys. Rev. Lett., 70 (1993) 1501; Herbut I. F., Phys. Rev. B, 57 (1998) 13729; Tešanović Z., Phys. Rev. B, 59 (1999) 6449.

[7] For applications to theories of the early universe, see Vilenkin A. and Shellard E. P. S., Cosmic Strings and Other Topological Defects (Cambridge University Press) 1994.

[8] Lawrie I., Nucl. Phys. B, 200 [FS 14] (1982) 1.

[9] Dasgupta C. and Halperin B. I., Phys. Rev. Lett., 47 (1981) 1556.

[10] Bartholomew J., Phys. Rev. B, 28 (1983) 5378.

[11] Herbut I. F. and TeŠanović Z., Phys. Rev. Lett., 76 (1996) 4588; 78 (1997) 980; Lawrie I., Phys. Rev. Lett., 78 (1997) 979.

[12] Kleinert H., Lett. Nuovo Cimento, 35 (1982) 405; Kiometzis M., Kleinert H. and Schakel A. M. J., Fortschr. Phys., 43 (1995) 697.

[13] Herbut I. F., J. Phys. A, 30 (1997) 423.

[14] Bergerhoff B., Freire F., Litim D. F., Lola S. and Wetterich C., Phys. Rev. B, 53 (1996) 5734.

[15] Olsson P. and Teitel S., Phys. Rev. Lett., 80 (1998) 1964.

[16] Nguyen A. K. and Sudbo A., Phys. Rev. B, 60 (1999) 15307; Sudbo A., Nguren A. K. and Hove J., preprint cond-mat/9907386. 
[17] For a review, see Folk R. and Holovatch Yu., in Correlations, Coherence, and Order, edited by Shopova D. V. and Uzunov D. I. (Kluwer) 1999; J. Phys. A, 29 (1996) 3409.

[18] Fisher M. P. A., Fisher D. S. and Huse D. A., Phys. Rev. B, 43 (1991) 130.

[19] Kamal S., Bonn D. A., Goldenfeld N., Hirschfeld P. J., Liang R. and Hardy W. N., Phys. Rev. Lett., 73 (1994) 1845.

[20] Yethiraj A. and Bechnoefer J., Phys. Rev. Lett., 84 (2000) 3642.

[21] See also, Chen J.-H., Lubensky T. C. and Nelson D. R., Phys. Rev. B, 17 (1978) 4274.

[22] Marynissen H., Thoen J. and van Dael W., Mol. Cryst. Liq. Cryst., 124 (1985) 195.

[23] Anisimov M. A., Mol. Cryst. Liq. Cryst. A, 162 (1988) 1.

[24] Lelidis I. and Durand G., Phys. Rev. Lett., 73 (1994) 672.

[25] RG and HLM results coincide at $x \approx 0.94$, when $t_{\mathrm{HLM}}=t_{0}$.

[26] See [11] also for a modified one-loop calculation that yields a flow diagram with correct topology.

[27] Lubensky T. C. and Chen J.-H., Phys. Rev. B, 17 (1978) 366.

[28] Because of well-known even-odd variations, the properties of the even-numbered chains are more similar to each other than are neighboring even- and odd-length molecules. 